\title{
Calibration of Ultrasonic Hardware for Enhanced Total Focusing Method Imaging
}

\author{
M. Ingram ${ }^{1 \dagger}$, A. Gachagan ${ }^{1}$, A. Nordon ${ }^{1}$, A. J. Mulholland ${ }^{2}$, and M. Hegarty ${ }^{3}$ \\ ${ }^{1}$ University of Strathclyde, Glasgow, UK \\ ${ }^{2}$ University of Bristol, Bristol, UK \\ ${ }^{3} \mathrm{BP}$ Chemicals Ltd, Hull, UK \\ ${ }^{\dagger}$ m.ingram@strath.ac.uk
}

\begin{abstract}
Experimental variation from ultrasonic hardware is one source of uncertainty in measured ultrasonic data. This uncertainty leads to a reduction in the accuracy of images generated from these ultrasonic data. In this paper, a quick, easy-to-use and robust methodology is proposed to reduce this uncertainty from images generated using the Total Focusing Method (TFM). Using a 128 element linear phased array, multiple Full Matrix Capture data sets of a planar reflection are used to characterise the experimental variation associated with each element index in the aperture. Following this, a methodology to decouple the time domain error associated with transmission and reception at each element index is presented. These time domain errors are then introduced into a simulated array model used to generate the two-way pressure profile from the array. The side-lobe to main-lobe energy ratio (SMER) and beam offset are used to quantify the impact of these measured time domain errors on the pressure profile. This analysis shows the SMER is raised by more than $6 \mathrm{~dB}$ and the beam is offset by more than $1 \mathrm{~mm}$ from its programmed focal position. This calibration methodology is then demon-
\end{abstract}

strated using a steel non-destructive testing sample with three side-drilled holes. The time delay errors from transmission and reception are introduced into the time-of-flight calculation for each ray path in the TFM. This results in an enhancement in the accuracy of the defect localisation in the TFM image.

\section{Introduction}

The Total Focusing Method (TFM) [1] has been at the forefront of much work in the ultrasonic imaging community and has been considered the gold standard ultrasonic imaging algorithm in NonDestructive Testing (NDT) [2]. The TFM constructs an image using the full matrix of aperture data acquired using an ultrasonic phased array, known as Full Matrix Capture (FMC) [1]. From the FMC data set it applies a delay and sum approach to focus the aperture data at every pixel in the image scene. For the TFM to generate an accurate representation of the image scene, it is critical that the timestamp for each A-Scan time domain signal in the aperture data is well defined. Indeed, it has been identified that time offsets are the most significant factor contributing to degradation of the TFM image scene with respect to error in the amplitude or phase of the aperture data [3].

The ultrasonic hardware, comprising of the phased 
array and the Phased Array Controller (PAC), are used to acquire FMC data. There is typically an underlying assumption that the ultrasonic hardware behave as designed, such that experimental variation deriving from the ultrasonic hardware is negligible. Under ideal conditions, for the lifespan of the ultrasonic hardware, all array elements should exhibit an identical impulse response function and all PAC channels should operate with an identical time delay. In practice, experimental variation from the ultrasonic hardware impacts on the timestamp of the aperture data creating a degree of uncertainty in the measured data. This is particularly acute if multiplexing array elements across multiple PAC channels is required to generate the FMC data set. In this paper, an experimental calibration methodology is proposed to remove this source of uncertainty from experimentally measured FMC data sets. In doing so, the accuracy of images generated using the TFM is enhanced.

Systematic error can be introduced into the FMC data during experimental measurement due to environmental, methodological or instrumental variation. For example, severe changes to the room temperature of a lab environment could impact on the density and bulk modulus of a target load, introducing variation in the speed of sound measured. Likewise, inconsistencies in gel couplant can introduce variation in the measured response across an array [4]. Most methodological variation cannot be completely removed from the experimental process, however, it can be minimised through careful experimental design. Variation in ultrasonic hardware could be introduced during manufacture. For example, the consistency of the matching and backing layers would impact on the measured sensitivity across the aperture. Such instrumental variation is typically minimised for hardware manufactured in batches greater than ten, where individual element performance must be within $1 \%$ of the design specification as set out by the International Organization for Standardization (ISO) 18563-1 [5].

In NDT, ultrasonic hardware calibration typically pertains to the efficacy of the array to steer and focus the ultrasonic beam [6], [3]. Therefore, in this context, under-performing hardware exhibit beam artefacts such as increased side-lobe activity, increased main-beam width or reduction in the main-beam energy level [6]. These beam artefacts lead to spurious signals being detected, increasing the uncertainty in the localisation of reflectors. As a result, calibration of ultrasonic hardware has typically focused on beam integrity rather than probe integrity [6].

Examples of phased array calibration have included investigation into the impact of inter-element amplitude [7] and phase variation [8] and the impact of reduced element sensitivity [9] on beam directivity. These methodologies have introduced a single criterion for the bulk array performance rather than investigating individual element performance. The nature of FMC means that no beamforming takes place during data acquisition, thus enabling variation between measured signals to be associated with individual element indices. Given that beamforming is the typical method of array deployment in industry, this also means there are few examples in the literature relating to ultrasonic hardware calibration using FMC. Indeed, the lack of calibration methods relevant to FMC has even been identified as a hindrance to its uptake by industry [10].

The impact of individual element performance on beam directivity was first investigated by Duxbury et al [6]. However, this was performed using simulated data sets, so the impact of variation due to the array being coupled to the PAC was not considered. The impact of peak time offset was investigated but the error associated with transmission and reception was not decoupled, instead a $50 \%$ error contribution from each process was assumed. In this paper, it is proposed that the peak time error resulting from transmission and reception at each element index can be decoupled and incorporated into the TFM algorithm.

This paper begins with an overview of the calibration materials and the data acquisition method used to generate an ultrasonic data set with a high degree of measurement certainty. Following this, the signal processing method used to extract information about the ultrasonic hardware from these measured data is described. This experimentally measured variation is then introduced into a simulated linear array model designed in Field II $[11,12]$ and the output of this model is used to quantify the impact of this variation. Results from an experimental data set are then 
discussed and the calibration methodology is demonstrated using a typical NDT steel test sample.

\section{Hardware Calibration Pro- cess}

\section{$2.1 \quad$ Materials}

The methodology has been designed to be quick, easy-to-use and robust, where the data acquisition procedure has been previously demonstrated by Ingram et al [13]. The underlying aspect of the calibration procedure was to acquire measured data corresponding to a known spatial position. This was achieved by recording the reflection from a planar surface using a linear array mounted to the opposite surface of a solid sample. An $n T_{x}=128$ element linear phased array (Vermon, Tours, France) with a 5 $\mathrm{MHz}$ designed centre frequency was deployed to acquire the ultrasonic data and the same array was used throughout the investigation to minimise instrumental variation. A crown glass block (Barr \& Stroud Ltd, Glasgow, UK) was chosen for the calibration sample because it permitted visual inspection, ensuring there was flaw-free path from the phased array to the back wall. The experimental set-up is outlined in Figure 1, where the straight line path length, $L$, to the back wall was $50 \mathrm{~mm}$. Surface roughness was not expected to have a significant impact on the path length to the back wall as even a $1 \%$ tolerance for the element position or pitch would result in a deviation in the path length three orders of magnitude greater than the micro-structure of the glass surface. There was also a high degree of certainty in the value of $L$ because the calibration sample was manufactured in bulk to specific dimensions. The sample was raised above the workbench to create an air load at the back wall, which increased the acoustic impedance mismatch generating a large reflection coefficient. To enhance the transmission of ultrasound into the calibration sample, water was used to couple the ultrasonic path between the array and the sample.

\subsection{Data Acquisition}

Using all available elements, FMC data of the back wall reflection were acquired five times in quick succession as this represented the typical deployment of the array during a single acquisition period. This also enabled the typical degree of experimental variation between FMC data sets to be captured. The array was not uncoupled from the PAC and the probe was kept in the same position on the surface of the calibration sample between FMC sample measurements to reduce variation due to sample heterogeneity. The acquisition process was approximately five minutes, including the set-up of apparatus, thus minimising the introduction of environmental variation into the measured data.

Following this, the signals corresponding to the two most reliable path lengths were extracted from the FMC data sets. Those signals to be extracted are demonstrated for a 16 element aperture in Figure 2, where they can be described as

I. The diagonal of the FMC matrix, corresponding to signals with same transmit and receive element indices and the vertical ray path for the propagating wave

II. The A-Scans with a receiving element index one greater than the diagonal, corresponding to transmission on element $n$ and reception on element $n+1$. Note, for transmission from element $n=n T_{x}$ this signal is not extracted as it does not exist.

The darker shaded squares in Figure 2 represent path length I and the lighter shaded squares represent path length II. The extraction process is performed for

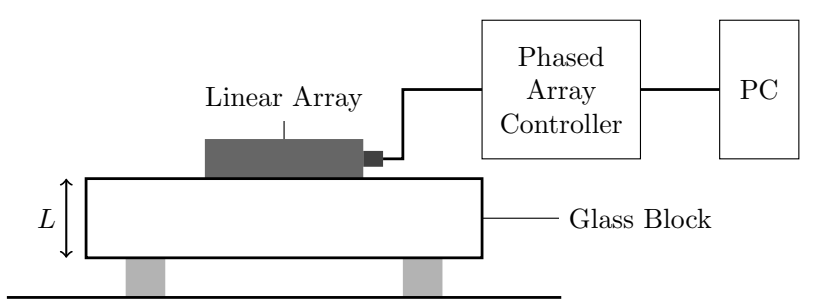

Figure 1: Apparatus for linear array calibration [13]. 


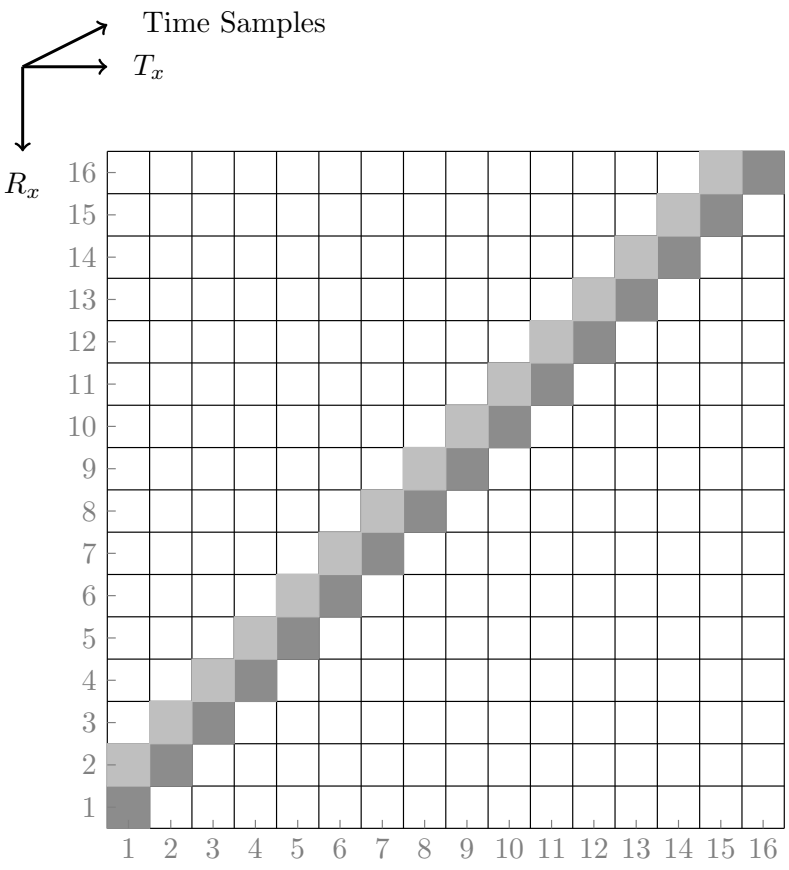

Figure 2: Schematic of FMC data set for a 16 element aperture, where each square represents an individual A-Scan and the shaded squares represent signals comprising the calibration data set.

each FMC data set and measurements between FMC data sets were only performed at the final stage of the signal processing methodology. Note, the A-Scans comprising the extracted FMC signals are collectively referred to as the calibration data set in the remainder of this paper.

\subsection{Peak Detection}

The signals in the calibration data set were then normalised prior to extraction of the peak time used for the calibration process. Each A-Scan time domain signal was mean-centred to remove any baseline offset that could be introduced by individual channels on the PAC. Next the artefact corresponding to the back wall reflection was windowed using a rectangular function with a value of zero everywhere except the time samples corresponding to the back wall echo where it has a value of one. Next the A-Scans were sent through a zero-phase Butterworth band-pass filter. For the purpose of this investigation the lower and upper bandstop frequencies were set to $\pm 1 \mathrm{MHz}$ of the probe's designed centre frequency. The filter design achieves a zero-phase shift by applying the filter once, reversing the order of the time samples, applying the same filter again and then reversing the time samples back to their initial order to recover the original signal phase with filtered amplitudes. Once the signals in the calibration data set were filtered, the next stage of the calibration process was the time domain detection of the back wall peak.

The peak detection methodology employed was similar to that developed by Namas et al [14]. For each A-Scan in the calibration data set, the Hilbert Transform was computed to reveal the in-phase, $S_{r}$, and quadrature, $S_{i}$, terms of the aperture data. From these, the envelope response, $E$ of the aperture data was computed

$$
E=\sqrt{S_{r}^{2}+S_{i}^{2}} .
$$

An example of the original A-Scan (post-filtering) and the envelope response is shown in Figure 3. The peak time was extracted from $E$ at the time sample corresponding to the maximum of the envelope and this was converted into units of seconds using the known sampling frequency.

\subsection{Speed of Sound Calculation}

To determine the speed of sound, $\nu$, in the glass calibration sample it was important to decouple variation due to the ultrasonic hardware from the calculation. This was achieved by measuring the Time-of-Flight (TOF) between the first and second back wall reflections, $t_{r e v}$, for A-Scans corresponding to the direct path to the back wall. If the time delay resulting from the PAC switching between transmit and receive modes is considered as a single event that is independent of the A-Scan length, the time difference between two subsequent reflections must be independent of ultrasonic hardware variation. Given that the distance travelled between the first two reflections is 




Figure 3: Time domain peak detection from envelope response.

known, $\nu$ can be determined,

$$
\nu=\frac{2 L}{t_{\text {rev }}} .
$$

Using the peak detection methodology outlined above, the time difference between the first two peak reflections was determined for the A-Scans corresponding to the FMC diagonal in each FMC replicate. From these values, the mean and standard deviation of $\nu$ were calculated to be $5973 \pm 85 \mathrm{~ms}^{-1}$ and these were assumed to be homogeneous throughout the glass calibration sample.

\subsection{Calibration Theory}

To calibrate the variation in the aperture data due to ultrasonic hardware variation, a peak time error, $t^{(n)}$, was established

$$
t^{(n)}=t_{e m}-t_{o b}
$$

where $t_{o b}$ is the observed peak time to the first back wall reflection for A-Scans in the calibration data set. The empirical TOF, $t_{e m}$, was equal to $t_{r e v}$ for AScans from the FMC diagonal. However, for the AScans with a receiving element index one greater than the diagonal, $t_{e m}$ was calculated by considering the element pitch, $p$

$$
t_{e m}=\frac{2}{\nu} \sqrt{L^{2}+\left(\frac{p}{2}\right)^{2}} .
$$

Two equations can be established that relate to the peak time error for each element index, $n$ :

I. $t_{1}^{(n)}$ - the peak time error associated with a direct path to the back wall for element index $n$, so transmitting on element $T_{x}=n$ and receiving on element $R_{x}=n$;

II. $t_{2}^{(n)}$ - the peak time error associated with a direct path to the back wall between two adjacent elements, so transmitting on element $T_{x}=n$ and receiving on element $R_{x}=n+1$.

Note, $T_{x}$ is the index of the element that is transmitting whereas $T_{x_{n}}$ is the time delay associated with the transmitting element index $n$. There are two unknowns for each element, the peak time error associated with transmission, $T_{x_{n}}$ and reception $R_{x_{n}}$. It was assumed that the total peak time error was the sum of the error from both the transmission and reception processes and so the following two equations can be derived

$$
\begin{aligned}
& t_{1}^{(n)}=T_{x_{n}}+R_{x_{n}} \\
& t_{2}^{(n)}=T_{x_{n}}+R_{x_{n+1}} .
\end{aligned}
$$

To solve for these parameters, the delay in transmission for the first element was assumed to be zero, such that $T_{x_{1}}=0$. This has the impact of normalising all time delays relative to a start time, $t_{0}$. In practice there will be a time delay between the PAC sending the instruction for element index 1 to fire and the element actually transmitting. However for FMC, all subsequent element transmission events are dependent on the previous element having already fired. Therefore, any delay in transmission of the first element is not dependent on a prior transmission delay so is irrelevant in the context of the timing of the overall data acquisition process. For the first transmitting element, $n=1$, Equations (5-6) give

$$
\begin{aligned}
& R_{x_{1}}=t_{1}^{(1)} \\
& R_{x_{2}}=t_{2}^{(1)} .
\end{aligned}
$$


Then the transmission delay for the subsequent element, can be determined by considering Equations (5-6) in the case $n=2$,

$$
\begin{aligned}
& t_{1}^{(2)}=T_{x_{2}}+R_{x_{2}} \\
& t_{2}^{(2)}=T_{x_{2}}+R_{x_{3}}
\end{aligned}
$$

where $R_{x_{2}}$ has already been determined in Equation (8). Therefore Equation (9) can be rearranged to solve for $T_{x_{2}}$ and following this, Equation (10) can be solved for $R_{x_{3}}$, so

$$
\begin{aligned}
& T_{x_{2}}=t_{1}^{(2)}-t_{2}^{(1)} \\
& R_{x_{3}}=t_{2}^{(2)}+t_{2}^{(1)}-t_{1}^{(2)} .
\end{aligned}
$$

This process is repeated for each transmitting element in the aperture and for each FMC replicate, resulting in an average peak time error for each element in both transmission and reception. So

$$
\begin{aligned}
& T_{x_{n}}=\sum_{m=2}^{n} t_{1}^{m}-\sum_{m=1}^{n-1} t_{2}^{m} \\
& R_{x_{n}}=\sum_{m=1}^{n-1} t_{2}^{m}-\sum_{m=2}^{n-1} t_{1}^{m} .
\end{aligned}
$$

Assuming that these values are representative of the error introduced by the hardware alone and must therefore be independent of sample heterogeneity, they can then be incorporated into the TFM algorithm by adding them to the TOF calculation for each ray path depending on which element is transmitting and receiving.

\subsection{Simulation of Pressure Field}

To quantify the impact of the measured time errors associated with transmission and reception determined from the calibration procedure, the pressure field was modelled in Field II $[11,12]$. The model was designed to closely match the design of the linear phased array used to acquire the calibration data set. The model consisted of a 128-element, $5 \mathrm{MHz}$ linear array with $0.7 \mathrm{~mm}$ element pitch. The array was coupled to steel with a speed of sound set to 6000 $\mathrm{ms}^{-1}$ and the sampling frequency set to $100 \mathrm{MHz}$.
The focal depth was set to $60 \mathrm{~mm}$ from the centre of the transducer and the focal laws were determined at azimuth angles of $0^{\circ}, 15^{\circ}, 30^{\circ}$ and $45^{\circ}$. The two-way pressure field was determined so that the measured peak time delays in both transmission and reception could be incorporated. The measured errors associated with transmission and reception were introduced into the model by adding the time error corresponding to each element to the focal laws.

The performance metrics used to quantify the impact of introducing the measured transmission and reception errors were the side-lobe to main-lobe energy ratio (SMER) and the beam offset. The SMER represents the integral of the beam intensity at the focal depth represented as a ratio between the energy contained within the main beam and the side lobes,

$$
S M E R=10 \log _{10}\left(\frac{\int_{-40 d B}^{-6 d B} I(x) d x}{\int_{-6 d B}^{0 d B} I(x) d x}\right),
$$

where $I(x)$ is the beam intensity and the lower sidelobe limit has been arbitrarily set to $-40 \mathrm{~dB}$. The SMER is the inverse of the signal-to-noise ratio, where a lower SMER value indicates a greater mainlobe intensity. The precise -6 and $-40 \mathrm{~dB}$ intensity positions were determined by performing linear interpolation on the pressure field data generated by Field II.

The beam offset is the absolute distance ( $\mathrm{mm}$ ) between the maximum pixel value in the main beam and the position of the focal point programmed by the focal law. Ideally, the beam offset should be zero and is included in this analysis to highlight how the accuracy of beamforming is dependent on error introduced by the hardware.

\section{Results and Discussion}

\subsection{Variation Deriving from the PAC}

The ultrasonic signal variation due to hardware degradation was investigated. The linear phased array was coupled to two different PACs. The newer PAC 1 was an FIToolbox (Diagnostic Sonar Ltd, Livingston, UK) whereas the older PAC 2 was a Dynaray 
(Zetec, Québec, Canada). Each were operated to acquire FMC data sets using the same transmit-receive channel configuration.

Under identical experimental conditions and using the methodology outlined in Section 2, the peak time to the back wall was recorded multiple times for the pulse-echo response from each element index. The results were plotted in Figure 4, where the error bars represent one standard deviation between FMC replicates. The results show that PAC 1 exhibited a greater degree of consistency across the array element indices than PAC 2. In addition, PAC 1 exhibited a smaller spread between the FMC replicates, shown by the smaller error bars. These results indicated that experimental data recorded from this linear phased array coupled to PAC 1 was more precise than when the same array was coupled to PAC 2.

The variation in element index performance associated with PAC 2 was not attributed to multiplexing of the 128 elements across the 32 channels available because no trends were observed between the channel and element indices in Figure 4. A possible source of the increased peak time spread for PAC 2 could be attributed to the recharge cycle of the capacitor connecting the element to the hardware electronics not being fully synchronised with the transmission process. This would mean the time taken to recharge the capacitor could vary and this variation could be passed downstream to the element transmission process, however, this is only speculative and could not be verified.

This highlights a constraint in terms of the applicability of this calibration methodology for TFM imaging. To accurately decouple the error associated with transmission and reception a precise understanding of the overall peak time error is required. Therefore, the spread between FMC replicates exhibited by PAC 2 resulted in the methodology being unable to accurately remove the measurement uncertainty due to the ultrasonic hardware from the TFM images. It is therefore recommended that $\mathrm{PAC} 2$ undergo a recalibration by the manufacturer to address this variation.

Overall, the results in Figure 4 highlight the degree of experimental variation that derives purely from the PAC, validating the need for this work. This, would suggest that future experimental FMC cali-

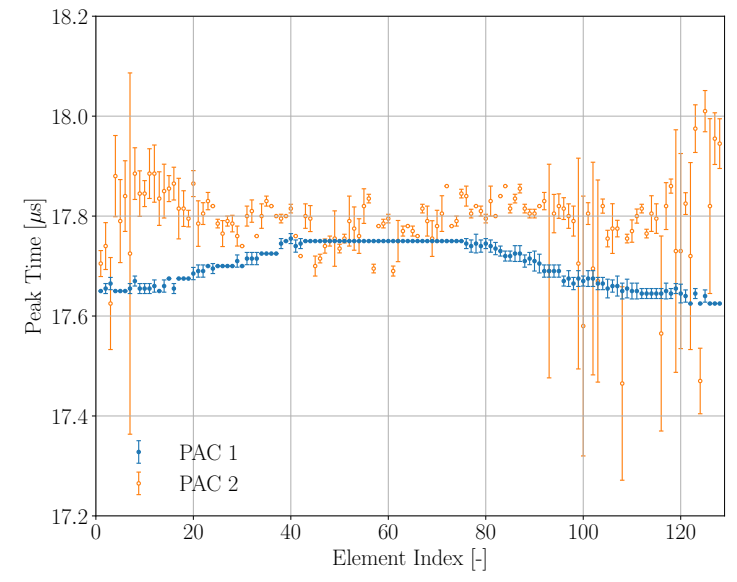

Figure 4: Variation in the peak time response of the first back wall reflection when the same phased array is coupled to two different PACs. The error bars represent one standard deviation between FMC replicates.

bration studies should always pertain to the overall phased array-PAC system rather than just the array itself. This is in agreement with standard industry practice whereby calibration on the combined system is laid out in ISO 18563-3 [15]. In addition, it is recommended that future studies aim to develop a criterion for an acceptable tolerance for the range of peak time responses with respect to their impact on TFM image deterioration.

\subsection{Decoupling of Transmit and Re- ceive Peak Time Error}

Using the calibration data set corresponding to the phased array coupled with PAC 1, the peak time error associated with transmission and reception was determined and plotted in Figure 5. It should be noted that these errors were independent of the start time delay typically used to crop the driving function from the A-Scan.

The error associated with transmission was approximately zero for all the element indices (recall that the 


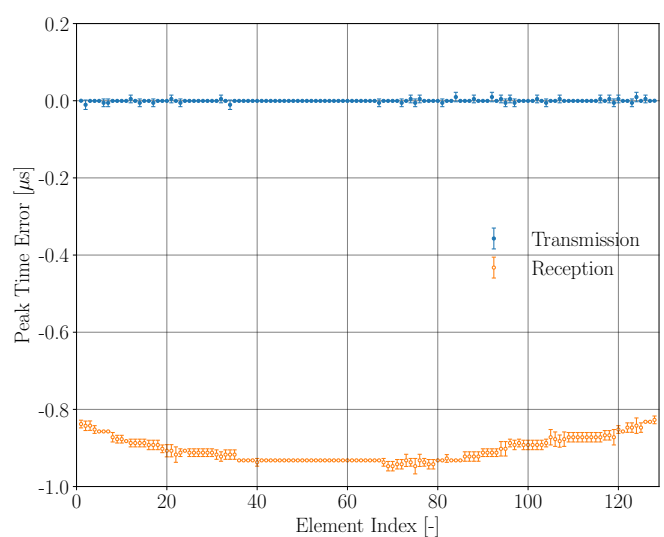

Figure 5: Decoupled transmit and receive peak time error to back wall for each element index.

transmission error for element index one was set to be exactly zero). The small error bars also indicate that the transmission error was consistent between FMC replicates. Where the transmission error exhibited a positive value, for example element index seventeen, this suggested that the error was due to the PAC firing slightly early. The error associated with reception was much larger in amplitude and was always negative. This suggested that there was a time delay associated with the PAC switching from transmit to receive mode. Similar to the transmission error, the small size of the error bars indicated there was a high degree of precision between the FMC replicates.

The average value of the total error in transmission and reception was $-0.96 \mu \mathrm{s}$, which was higher than the threshold of $\pm 0.5 \mu$ s determined by Zhang et al [3] that led to TFM image degradation. Therefore, it was concluded the errors demonstrated here reduced the accuracy of the focussing ability of the TFM algorithm.

\subsection{Impact of Peak Time Error on Simulated Pressure Field}

The measured values of peak time error associated with transmission and reception were incorporated into the focal laws used during determination of the simulated pressure field. Note, in the simulated data, (a)

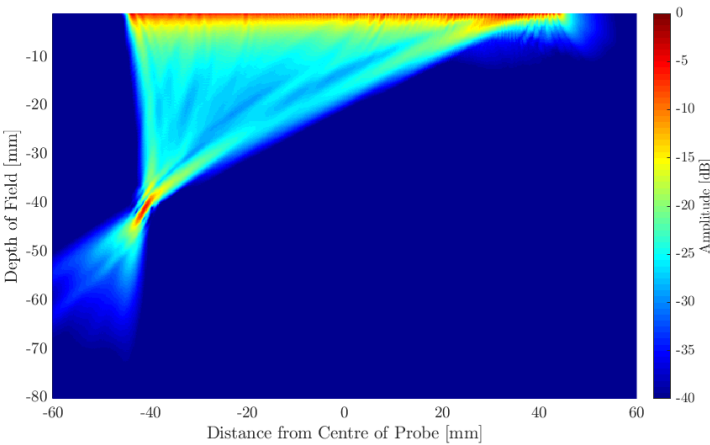

(b)



Figure 6: Pressure field for beam steered at $45^{\circ}$ in azimuth direction and at a focal depth of $60 \mathrm{~mm}$ (a) with measured time errors introduced into the focal law (b) standard focal law.

$T_{x_{n}}$ and $R_{x_{n}}$ do not exist as all element indices exhibit identical behaviour. Therefore, $T_{x_{n}}$ and $R_{x_{n}}$ needed to be introduced to the focal laws to observe the same phenomenon observed with experimental data. An example is shown in Figure 6(a), where the measured peak time errors have been added to the focal law corresponding to a $45^{\circ}$ azimuth angle. Whereas, in Figure 6(b), the pressure field is restored to its correct state by not introducing $T_{x_{n}}$ and $R_{x_{n}}$ to the focal law.

From Figure 6 it is observed that the pressure field is slightly distorted but not completely compromised by introducing these measured time delay errors. Inspection of the SMER values in Table 1 shows that 
introducing the measured $T_{x_{n}}$ and $R_{x_{n}}$ values lowers the SMER by an average of $7.9 \mathrm{~dB}$ across the azimuth angles investigated. This means that over $75 \%$ of the power injected into the beam, relative to the calibrated system, is spread into the side-lobes and not directed towards the main beam, reducing the accuracy of the focused beam. Indeed, it is observed from Table 2 that the average beam offset is $1.76 \mathrm{~mm}$ when the time delays are introduced and this corresponds to approximately $1.8 \lambda$ for this system. Therefore, if the positioning of a defect needs to be accurate on this length scale, errors introduced by the hardware alone could compromise such an investigation.

Table 1: SMER measured from simulated pressure field where time delay errors were introduced into the model to observe the same experimental response.

\begin{tabular}{ccc}
\hline Azimuth & \multicolumn{2}{c}{ SMER [dB] } \\
\cline { 2 - 3 } Angle & Errors Introduced & Without Errors \\
\hline $0^{\circ}$ & -2.75 & -9.62 \\
$15^{\circ}$ & -2.13 & -9.94 \\
$30^{\circ}$ & -1.46 & -9.70 \\
$45^{\circ}$ & -0.48 & -9.35 \\
\hline
\end{tabular}

Table 2: Beam offset measured from simulated pressure field where time delay errors were introduced into the model to observe the same experimental response.

\begin{tabular}{ccc}
\hline Azimuth & \multicolumn{2}{c}{ Beam Offset $[\mathrm{mm}]$} \\
\cline { 2 - 3 } Angle & Errors Introduced & Without Errors \\
\hline $0^{\circ}$ & 1.39 & 0.40 \\
$15^{\circ}$ & 1.44 & 0.38 \\
$30^{\circ}$ & 1.86 & 0.43 \\
$45^{\circ}$ & 1.71 & 0.72 \\
\hline
\end{tabular}

\subsection{Experimental Application of Cal- ibration Procedure}

To demonstrate the application of the calibration methodology, the phased array coupled with PAC 1 were used to acquire an FMC data set from a typical NDT steel test block. A schematic of the test sample is shown in Figure 7, where there were three 3 $\mathrm{mm}$ diameter side-drilled holes $(\mathrm{SDH})$ at increasing depths. The probe was centred over the three holes and an FMC data set was acquired using all available elements. The A-Scans in this FMC data set were sent through a zero-phase Butterworth bandpass filter with upper and lower bandstop frequencies of $\pm 3 \mathrm{MHz}$ to reduce the impact of noise on the final image. The speed of sound in the test sample was determined to be $5873 \pm 86 \mathrm{~ms}^{-1}$ using the procedure outlined in Section 2.4 using part of the sample that was flaw-free.

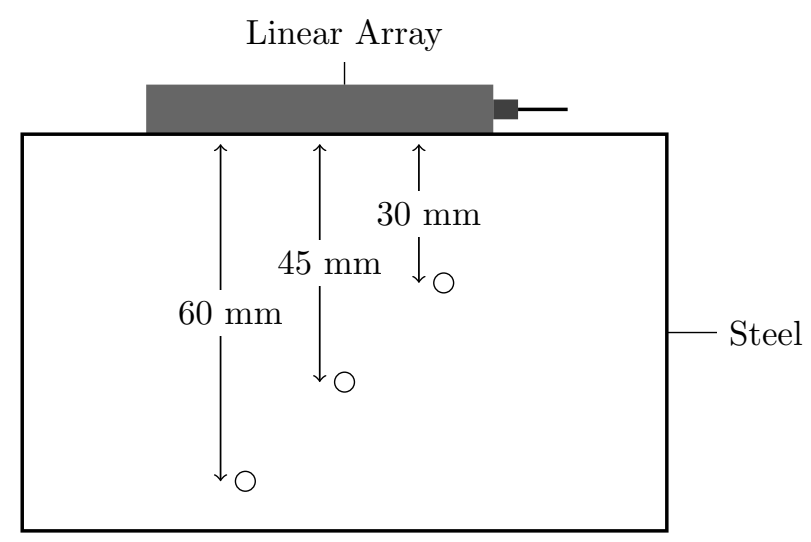

Figure 7: Schematic of steel test block.

The image scene was constructed using the TFM and the envelope response was converted to decibel scale for displaying the images. Figure 8(a) represents the image scene without any calibration for the transmission or reception errors. For all three SDHs the depth of the focal point has been positioned below the true location. The image resulting from the introduction of the transmission and reception errors to the TFM is shown in Figure 8(b). The result of the calibration procedure has increased the size of the reflectors and shifted their positions to be closer to their true locations. This complements the findings recorded in Table 2 by showing the beam offset can reduce the accuracy of defect localisation. Note, given that the calibration data for PAC 1 exhibited a 
precise and accurate peak time to the back wall of the calibration sample, the small enhancement observed in the TFM images here is expected. On the other hand, if the calibration data were to exhibit precise yet inaccurate peak time values, the enhancement in TFM image accuracy is expected to be greater.

(a)



(b)

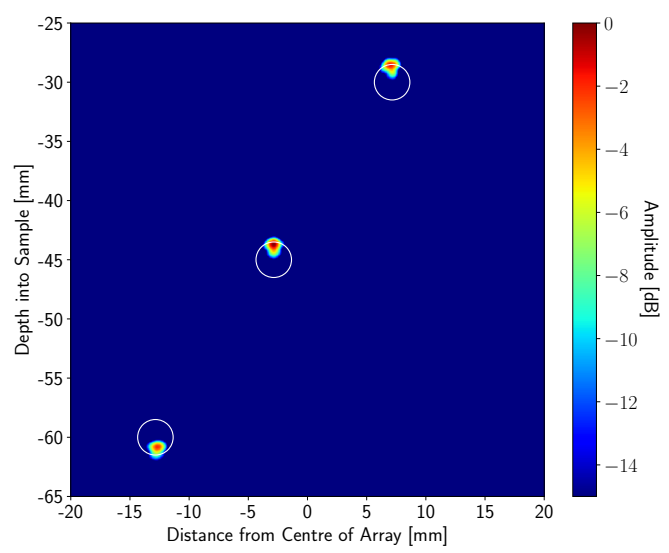

Figure 8: TFM image of NDT steel test block with three SDHs, where white circles represent the true location of the reflectors, (a) standard image and (b) calibrated image.

In addition, the shape of the reflectors in the calibrated image more closely represents the shape of the reflecting surface. This was especially true for the reflector positioned $30 \mathrm{~mm}$ from the probe. Upon closer inspection of this region in Figures 9(a) and 9(b), it was observed that the TFM algorithm more accurately mapped the curvature of the surface of the reflector as a result of the calibration procedure.

(a)

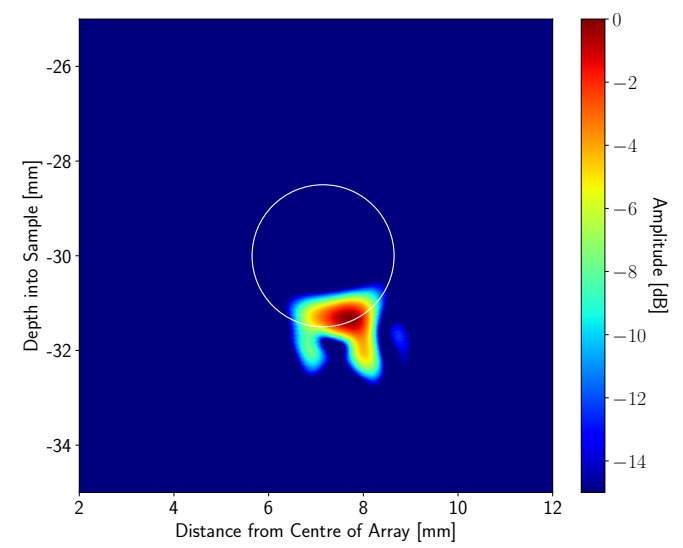

(b)

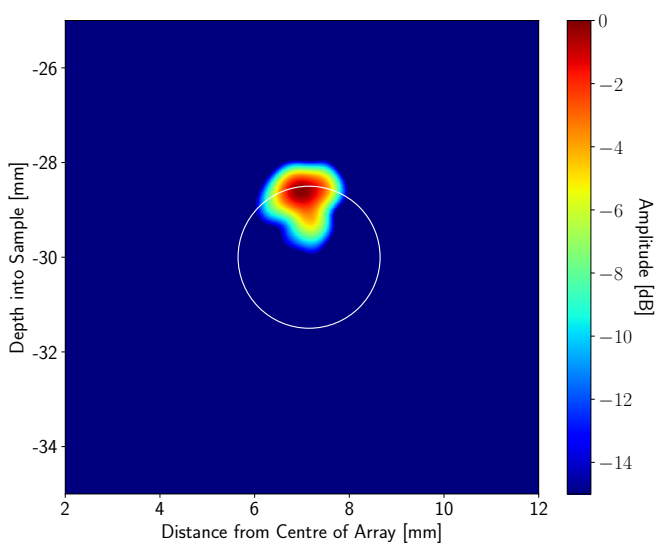

Figure 9: TFM image of SDH at $30 \mathrm{~mm}$ into NDT steel test block, where white circles represent the true location of the reflectors, (a) standard image and (b) calibrated image.

In practice the proposed calibration methodology is applicable to any test sample because it has been developed to isolate, as much as possible, the variation deriving from the hardware system. This also 
means that the transmit and receive time delays relate specifically to the array-PAC combined system, limiting its applicability to other systems. The accuracy of this methodology is mostly dependent on the empirical or reference TOF, $t_{e m}$, determined in Equation 4. Given that the glass block was manufactured in bulk to specific dimensions, there was a high certainty in the value of $L$. In addition, any uncertainty introduced by surface roughness was considered negligible given the error in the value of $p$ was considered to be several orders of magnitude greater. Moreover, the value of $p$ was set during the transducer manufacture and so was considered a constant value based on the transducer's technical report. In light of this, the main source of uncertainty is in the measurement of $\nu$. Indeed, the spread of $\nu$ values indicates a degree of heterogeneity in the glass calibration sample and/or variation in transduction efficiency between measurements could exist. These factors are typically difficult to control or modify and so it should be noted that the robustness of the methodology is sensitive to the measured speed of sound in the calibration sample.

\section{Conclusions}

An experimental methodology has been presented for the time domain calibration of linear phased arrays coupled with phased array controllers. Using FMC of a plane reflection, the integrity of the ultrasonic hardware has been assessed at each individual element index in the aperture. The time domain error associated with transmission and reception from each element index has been decoupled. When these time errors were introduced into the focal laws used to determine the pressure field of a simulated array, the side-lobe to main-lobe energy ratio was raised by more than $6 \mathrm{~dB}$ and the main beam was offset by more than $1 \mathrm{~mm}$. When these time errors were incorporated into the TFM algorithm the accuracy of the image was enhanced. This will in turn lead to more accurate localisation of flaws in NDT. In addition, it was identified that the time domain error was dependent on the PAC used to drive the array. Therefore, it is suggested that experimental calibration of phased arrays should always pertain to the overall array-PAC system and not just the array itself.

\section{Acknowledgements}

The authors would like to thank the Engineering and Physical Sciences Research Council (grant number EPM5076471) and BP Chemicals Limited for their support and industrial direction.

\section{References}

[1] C. Holmes, B. Drinkwater, and P. Wilcox. Post-processing of the full matrix of ultrasonic transmit-receive array data for non-destructive evaluation. NDT \& E International, 38(8):701 711, 2005.

[2] Kai E Thomenius. Evolution of ultrasound beamformers. In Ultrasonics Symposium, 1996. Proceedings, volume 2, pages 1615-1622. IEEE, 1996.

[3] J. Zhang, B. Drinkwater, and P. Wilcox. Effects of array transducer inconsistencies on total focusing method imaging performance. NDT \& $\mathrm{E}$ International, 44(4):361-368, 2011.

[4] David Duxbury. Calibration and control of advanced ultrasonic array technology. 2013.

[5] Non-destructive testing: Characterization and verification of ultrasonic phased array equipment - Part 1: Instruments. Standard, International Organization for Standardization, Geneva, CH, July 2017.

[6] D. Duxbury, J. Russell, and M. Lowe. The effect of variation in phased array element performance for non-destructive evaluation (NDE). Ultrasonics, 53(6):1065 - 1078, 2013.

[7] C.T. Lancée, J.M. Vissers, S. Mientki, C.M. Ligtvoet, and N. Bom. Influence of amplitude errors on beam-steered phased arrays. Ultrasonics, 25(3):147-153, 1987. 
[8] C.T. Lancée, J.M. Vissers, S. Mientki, C.M. Ligtvoet, and N. Bom. Influence of phase errors on beam-steered phased arrays. Ultrasonics, 25(3):154-159, 1987.

[9] C. Nageswaran. Coping with failed elements on an array: a modelling approach to the technical justification. Insight-Non-Destructive Testing and Condition Monitoring, 52(7):372-379, 2010.

[10] S. Mosey, M. Sutcliffe, M. Weston, B. KnightGregson, and D. Liaptsis. Development and implementation of calibration process for full matrix capture. In Proceedings of the 53rd Annual Conference of The British Institute of NonDestructive Testing. Manchester: BINDT, 2014.

[11] Jørgen Arendt Jensen. Field: A program for simulating ultrasound systems. In 10TH Nordic Baltic Conference on Biomedical Imaging, volume 4, pages 351-353. Citeseer, 1996.

[12] Jørgen Arendt Jensen and Niels Bruun Svendsen. Calculation of pressure fields from arbitrarily shaped, apodized, and excited ultrasound transducers. IEEE transactions on ultrasonics, ferroelectrics, and frequency control, 39(2):262267, 1992.

[13] M Ingram, A Gachagan, AJ. Mulholland, A Nordon, J Dziewierz, M Hegarty, and E Becker. Calibration of ultrasonic phased arrays for industrial applications. In Proceedings of IEEE Sensors, pages 1518-1520. IEEE, 2017.

[14] T. Namas and M. Dogruel. A feasible and accurate technique for determining the time-of-flight in ultrasonic distance measurements. In $E L$ $M A R, 2008$. 50th International Symposium, volume 1, pages 337-340. IEEE, 2008.

[15] Non-destructive testing: Characterization and verification of ultrasonic phased array equipment - Part 3: Combined Systems. Standard, International Organization for Standardization, Geneva, CH, July 2017. 